\title{
La etapa de los bienes globales en salud, una perspectiva nacional
}

\author{
José Ángel Córdova-Villalobos, MC, M Esp, M en Admón Púb. ${ }^{(1)}$
}

\author{
Córdova-Villalobos JA. \\ La etapa de los bienes globales en salud, \\ una perspectiva nacional. \\ Salud Publica Mex 2008;50 supl 3:S309-S3 I4.
}

\begin{abstract}
Resumen
Los efectos adversos del tabaquismo sobre la salud han sido ampliamente reconocidos desde tiempo atrás. A pesar de todo, el tabaquismo no parece disminuir, con las inevitables consecuencias sobre la morbilidad y la mortalidad de millones de personas. Tan sólo en este año la OMS calcula que cinco millones de personas morirán por esta causa. En México, $26.2 \%$ de la población entre 15 y 65 años fuma con regularidad y el consumo se incrementa entre los jóvenes y las mujeres. La OMS adoptó el Convenio Marco para el Control del Tabaco, ratificado por México, lo que llevó a la creación de la Ley General para el Control del Tabaco, un importante hito en la política sanitaria mexicana, que próximamente será promulgada y que se espera tendrá un efecto notable en la contención de la epidemia de tabaquismo en el país.
\end{abstract}

Palabras clave: tabaco; politicas públicas; derechos civiles; México

\section{Córdova-Villalobos JA.}

The age of public goods in health, a national perspective.

Salud Publica Mex 2008;50 suppl 3:S309-S3 I 4.

\section{Abstract}

Adverse health effects from smoking have been widely known for a long time. Nevertheless, tobacco use does not appear to decrease, and with it the inevitable consequences on morbidity and mortality for millions of people continue. This year alone, the $\mathrm{WHO}$ calculates that five million people will die from tobacco use. In Mexico, $26.2 \%$ of the population between the ages of 15 and 65 smoke regularly and consumption increases among youth and women. The WHO adopted the Framework Convention on Tobacco Control, ratified by Mexico, which brought about the General Law for Tobacco Control, an important milestone in Mexican sanitary policy. This law will soon be in force and is expected to have a notable effect on containing the smoking epidemic in the country.

Key words: tobacco; public policies; civil rights; Mexico

\section{La epidemia}

Se sabe desde hace muchos años: el tabaco es un producto que, pese a ser legal, es adictivo y mata entre un tercio y la mitad de sus consumidores. Millones de fumadores sufrirán diferentes enfermedades causadas por los diferentes componentes presentes en el humo de tabaco y acortarán al menos 15 años su esperanza de vida.
El tabaco y sus productos afectan no sólo a quienes los consumen directamente, sino también a un incalculable número de no fumadores que tiene que respirar el aire contaminado con el humo del tabaco de los fumadores activos. Sus consecuencias sanitarias han sido bien caracterizadas por medio de innumerables estudios científicos y actualmente son aceptadas y bien conocidas. Además de las enfermedades provocadas por

(I) Secretario de Salud, México. 
el consumo directo de tabaco y las causadas por esta exposición pasiva, la dependencia del tabaco -la adicción a la nicotina- es también una enfermedad y que, como tal, priva de la libertad a quien la padece y lo transforma en un sujeto dependiente de una sustancia.

Entre los aspectos más importantes derivados del consumo de tabaco se encuentra esta doble morbilidad, por un lado, la que deriva del efecto adverso de las sustancias y sus ingredientes en el organismo humano, tanto de las personas que fuman cuanto en aquellos considerados como "fumadores pasivos" que inhalan involuntariamente, por razones sociales o laborales, el humo ajeno; y por el otro, la inducción que el consumo del tabaco hace para iniciar el consumo de otras sustancias adictivas, como el alcohol, la mariguana, la cocaína, las metanfetaminas y otras. El tabaco es la puerta de entrada a otras adicciones; un niño que fuma tiene 13 veces más posibilidades que otro que no lo hace, de experimentar en el corto plazo con otras drogas y hacerse adicto a ellas, de ahí la doble importancia de controlar esta peligrosa sustancia. Al disminuir el acceso de los niños y jóvenes al tabaco, habremos disminuido también la curiosidad por experimentar con otras drogas.

En todo el mundo se fuman cada día la difícilmente imaginable cantidad de más de 15 mil millones de cigarrillos. Se estima que para el año 2025, el número de fumadores alcanzará cerca de 1.6 mil millones, por encima de los 1.3 mil millones actuales y que, de ellos, 650 millones morirán en los siguientes años, dejando de producir y de disfrutar de, por lo menos, 20 años de vida saludable. Estas muertes prematuras tienen un costo social y económico extraordinariamente alto para la sociedad.

El crecimiento global de la población que fuma y el incremento desproporcionado en el número de fumadores que se ha registrado en las últimas décadas, se han basado en la manera como la industria tabacalera responde a su propio dilema, ¿cómo seguir vendiendo un producto altamente adictivo que aniquila a la mitad de sus usuarios habituales? La respuesta ha sido muy clara, asociar la imagen del fumador con valores positivos que nada tienen que ver con la característica primordial del producto, a través del uso de situaciones atractivas ligadas, paradójicamente, al poder, la belleza, la salud y la vida.

La etapa que ha tocado vivir a las presentes generaciones está dominada por la globalización de la vida y en salud no podría ser de otro modo. La Organización Mundial de la Salud (OMS) ha estimado que debido al consumo de cigarrillos tan sólo en este año morirán prematuramente en el mundo más de 5 millones de personas como consecuencia del consumo de tabaco y que, de seguir con esta tendencia, para 2020 esta cifra podría llegar a los 10 millones de personas, 70\% de las cuales vivirán en países en vías de desarrollo. Países en los que la industria ha encontrado marcos regulatorios débiles y sociedades desprevenidas y donde la industria ha logrado promover el consumo de tabaco intensamente, en particular entre los jóvenes y las mujeres.

Cómo será de grave esta epidemia que, de acuerdo con esta misma institución, se ha estimado que mundialmente el tabaquismo, por sí solo, es responsable de más muertes que el VIH-SIDA, el alcohol, las lesiones por accidentes, los homicidios, los suicidios, los desastres por fuego y la tuberculosis, juntos.

Trasladadas algunas de estas cifras a México, datos recientes indican que diariamente mueren 163 personas debido a las complicaciones ocasionadas por fumar; del total de poco más de 16 millones de personas que lo hacen, los que se suman a los cerca de 38 millones de mexicanos expuestos involuntariamente o por razones ocupacionales al humo de tabaco ajeno $(70.52 \%$ de mujeres y $29.48 \%$ de hombres).

Datos recientes de la encuesta mundial de tabaquismo en jóvenes, en su capítulo mexicano, indican que cerca de $20 \%$ de los estudiantes de secundaria ya son fumadores activos, 1 de cada 10 adolescentes de 12 a 17 años lo hace con regularidad y la relación por género ha llegado a ser ya de 1 a 1. El 7\% de los jóvenes fumadores lo hacen por primera vez a los 10 años o menos, $45 \%$ inicia el consumo entre 11 y 14 años y $48 \%$ entre 15 y 17 años.

Esto significa que en México, $26.2 \%$ de la población entre 15 y 65 años fuma, cifra que comparada con el resto del planeta (30\%): Alemania, $(27.5 \%)$, Reino Unido $(27 \%)$, Italia (27.5\%), Dinamarca (27\%), Rusia (45\%) o Turquía (40\%), podría parecer inferior a estos países, pero que comparada con países como Suecia $(20.5 \%)$, por ejemplo, resulta muy superior.

Estas cifras conducen a una conclusión que, no por elemental, deja de ser grave: la expresión que esta epidemia mundial tiene en México es preocupante y sus tendencias a continuar creciendo, expresadas en una disminución de la edad promedio del consumo, el incremento del consumo experimental entre menores de edad y el incremento de la prevalencia de mujeres fumadoras, deben alertarnos. Especialmente, la cifra que supone una relación de género de 1 a 1 , ya que sugiere fuertemente que en el corto plazo se avecina una epidemia de importante magnitud entre las mujeres.

\section{Respuestas a problemas globales}

En la evolución de esta epidemia ha habido voces razonables que han permitido que la epidemia no pase desapercibida por tantos años, y que sea enfrentada 
de manera global a través de la tutela de un bien que también lo es, superando los intereses particulares o parciales representados por la industria. De esta forma, el 21 de mayo de 2003 -un día histórico para la salud pública mundial- la 56a Asamblea Mundial de la Salud adoptó, por unanimidad de los 192 Estados Miembros de la OMS, el primer tratado mundial de salud pública de la historia, el Convenio Marco para el Control del Tabaco (CMCT).

Este tratado ha sido firmado y ratificado, hasta ahora, por 168 países y México, como primer país de Latinoamérica en ratificarlo en mayo de 2004, ingresó con él en una nueva etapa de la salud, marcada por el compromiso de instrumentarlo en toda la nación y reforzar las acciones locales para el control del tabaco. Este compromiso es de gran importancia por tratarse de una de las agendas de salud pública moderna de mayor beneficio e impacto potencial en salud.

Los principios rectores del Convenio son:

a) Acceso de toda la población a la información sobre las consecuencias sanitarias, la naturaleza adictiva y el peligro mortal por el consumo y la exposición al humo de tabaco.

b) Establecimiento de compromisos locales suficientemente firmes como para instituir respuestas coordinadas y respaldar medidas multisectoriales integrales.

c) Ampliación de la cooperación internacional, particularmente de la transferencia de tecnologías, conocimientos y asistencia financiera.

d) Prevención de las enfermedades, la discapacidad y la mortalidad prematura causadas por el tabaco.

e) Promoción de la aceptación de la responsabilidad, penal y civil, inclusive la compensación cuando proceda.

f) Asistencia técnica y financiera para aquellos cuyos medios de vida se afecten por los programas de control del tabaco.

g) Promoción de la participación de la sociedad civil como medida para conseguir el objetivo del Convenio y de sus protocolos.

En esencia, el tratado invita a que los países que lo han firmado impongan restricciones a la publicidad, el patrocinio y la promoción del tabaco; adopten un nuevo envasado y etiquetado de los productos de tabaco con información realista del peligro del consumo; determinen controles para proteger a los no fumadores con el establecimiento de ambientes $100 \%$ libres de humo en espacios cerrados, establecimiento de políticas fiscales que desincentiven el consumo de tabaco y fortalecer la legislación para combatir enérgicamente el contrabando de tabaco, entre otras medidas

Acciones equivalentes se venían aplicando en muchos países, México entre ellos, desde que se estableció la Ley General de Salud (1986) y con modificaciones importantes en 1994 y 2000 (protección a no fumadores e identificación de edificios federales como libres de humo de tabaco con áreas restringidas para ello).

Sin embargo, las características primordiales de estas medidas han sido su parcialidad y su diversidad y, por ello, su impacto ha sido irregular en la salud de las poblaciones. Por citar un ejemplo, todavía hoy existen en México 20 leyes estatales para la protección de los no fumadores diferentes entre sí; 10 reglamentos locales sobre la materia que son distintos y en otras dos entidades las regulaciones antitabaco se hacen a través de otros ordenamientos. Lo que deja muchos espacios de oportunidad para que las compañías tabacaleras violen las disposiciones y continúen promocionando sus productos. Dejan también espacios ambiguos en lo que se refiere a la protección a no fumadores y por consecuencia una exposición amplia de los no fumadores al humo de tabaco ajeno.

La competencia de la autoridad sanitaria en la materia había sido asumida de manera prácticamente concurrente y desde hace quince años existe una regulación centralizada de la publicidad de los productos de tabaco que establece la prohibición de hacerlo en medios como el cine, la radio y la televisión, a cambio de permitirlo a través de anuncios espectaculares y otros medios alternativos, las revistas y otros vehículos similares. Una situación análoga puede decirse de la prohibición de la venta a menores y otras regulaciones.

Sin embargo, en conjunto, es claro que todas estas medidas han sido insuficientes para contener la epidemia de tabaquismo y, por el contrario, como ya se mencionó, no han atajado el crecimiento de la prevalencia, lo que plantea un desafío sanitario de primer orden, al cual se respondió durante los dos primeros años de la ratificación del Convenio por parte de México, frenando la instrumentación de algunas de las acciones que derivaban del mismo y exponiendo el cumplimiento de los compromisos, a cambio de acordar con la industria tabacalera la constitución de un fondo para gastos catastróficos en salud en condiciones que así lo indujeron, a cambio de aportaciones económicas voluntarias de las empresas tabacaleras a ese fondo, a manera de donaciones.

La actual administración sanitaria mexicana nulificó estos acuerdos y optó por retomar de manera decidida la aplicación del CMCT y avanzar en el cumplimiento de nuestros compromisos con él, en los plazos establecidos, 
fortaleciendo las instancias administrativas designadas para ello.

En el periodo reciente, la confluencia en esta tarea de iniciativas de organizaciones de la sociedad civil y de fundaciones que coinciden en las finalidades con las de la autoridad responsable de la protección de la salud de las mexicanas y los mexicanos ha sido un ingrediente que debe destacarse porque ha acompañado el esfuerzo de personas e instituciones perseverantes en la reducción de los daños atribuibles al tabaquismo y en la contención de la epidemia.

\section{Instrumentos y herramientas para impulsar el CMCT en México}

De acuerdo con el Artículo 133 de nuestra Constitución, todo tratado internacional ratificado por el Senado adquiere rango de ley, y por ello, desde la LIX Legislatura se habían impulsado iniciativas para modificar favorablemente diversos ordenamientos legales que permitieran enfrentar, con mejores herramientas, las tareas asumidas por México al ratificar el CMCT.

Lejos de inducir modificaciones parciales a diversos ordenamientos, una veintena de diputados y senadores de las comisiones de Salud de la actual Legislatura, justo en el primer aniversario de su instalación, en conferencia promovieron una Iniciativa para establecer una Ley General para el Control del Tabaco (LGCT) que asumiera, en un solo cuerpo legal y su reglamento, todo el control sanitario de los productos del tabaco, así como su importación y la protección contra la exposición al humo de tabaco, cuyo ejemplar proceso legislativo se dio entre agosto de 2007 y febrero de 2008.

De manera paralela, la Asamblea de Representantes del Distrito Federal legisló en torno a una Ley de Protección a los No Fumadores para la capital, y las modificaciones a ordenamientos relacionados, como el de Establecimientos Mercantiles, fundamentalmente para establecer como ambientes $100 \%$ libres de humo de tabaco todos los espacios públicos cerrados, sin excepción.

La autoridad sanitaria federal no sólo vio en este tipo de instrumentos una veta riquísima para el cumplimiento de sus atribuciones, sino que avaló su contenido y, conjuntamente con el Legislativo, apoyó decididamente su promoción, abonando el terreno para favorecer su institucionalización como parte de la política sanitaria nacional.

Como autoridad apoyamos la iniciativa en su momento porque nos irradiaba un saldo positivo respecto de algunos aspectos fundamentales en la protección de la salud de las mexicanas y los mexicanos frente al tabaco y sus productos, entre otros:
- La garantía para la protección de la salud de todas las personas, fumadoras o no fumadoras, hombres o mujeres, grandes o pequeños, pero especialmente de quienes resultarían más perjudicados con la exposición al humo de tabaco (niñas, niños, mujeres embarazadas, enfermos del aparato respiratorio y adultos mayores).

- El reconocimiento del control del tabaco como materia de utilidad pública e interés social y la identificación de las atribuciones y facultades en la materia.

- La creación de un medio para que México pueda cumplir de manera integral con compromisos internacionales, contraídos al ratificar el CMCT de la OMS por parte del Senado.

- El establecimiento para las compañías tabacaleras de la obligación de entregar información sobre el contenido de sus productos para que ésta se haga pública y se cumpla con el derecho ciudadano a la información.

- La regulación clara de los lugares donde se puede vender productos de tabaco y la forma de hacerlo, establecer que las niñas y los niños no pueden exponerse a la publicidad que sólo se permite dirigir a los adultos.

- El establecimiento de espacios 100\% libres de humo de tabaco en cualquier espacio público cerrado y autorización del consumo en espacios abiertos o en zonas exclusivas aisladas, adaptadas o acondicionadas ex profeso para ello (cerrados de suelo a techo, con sistemas de extracción y en lugares que no sean lugares de tránsito), lo que garantiza la libertad de quienes fuman para que lo hagan sin perjudicar a terceros. El polémico "derecho" de los fumadores quedaba así cubierto de la misma forma que el derecho de los no fumadores a respirar un aire limpio y proteger su salud.

- La inclusión obligatoria de leyendas y-por primera vez en México-de pictogramas o imágenes sanitarias de advertencia, rotativas y claras que obligan a la industria tabacalera a eliminar términos engañosos o confusos y a advertir a los consumidores sobre los peligros que entraña utilizar productos de tabaco.

- El establecimiento claro de sanciones administrativas para quienes violen sus disposiciones, así como los mecanismos para ejecutarlas e identificación de las autoridades y procedimientos para ello.

- La promoción de la participación ciudadana para aplicar y vigilar su aplicación y reconocimiento de la figura de la denuncia ciudadana como medio de hacerlo.

- La identificación de la Secretaría de Salud como la autoridad responsable para su aplicación, así como 
para hacer los reglamentos correspondientes y establecimiento de la obligación para que la propia Secretaría tenga en operación una línea telefónica para recibir denuncias y ofrecer servicios de cesación para fumadores que desean abandonar esta adicción.

- La protección de los derechos humanos de los ciudadanos al ser aplicada.

La sensibilidad de los legisladores, diputados y senadores, encontró un contexto favorable para la aprobación de la iniciativa y el 6 de diciembre de 2007 y el 26 de febrero de 2008 marcaron un hito al ser aprobada por una amplia mayoría en la Cámara de Diputados y en la de Senadores, respectivamente.

Siguiendo con la lógica de este proceso, estamos esperando la publicación de la ley aprobada, la cual será vigente a los 90 días de ello y, paralelamente, la autoridad sanitaria trabaja en la elaboración del reglamento respectivo, en el cual se incluirán las medidas de protección y las medidas que son objeto de regulación, las cuales comenzarán a 90 días después de que se publique dicho documento.

Por su parte, la Ley para la Protección de los No Fumadores del Distrito Federal fue más estricta en lo tocante al establecimiento de espacios libres de humo que la Ley General, y su aprobación fue un acierto, fue recibida con gran entusiasmo, en medio de una discusión social muy ilustrativa del grado de preocupación y participación de la población en el tema. Llamó gratamente la atención -en el proceso de su implementación- la actitud y colaboración de la ciudadanía para acatar sus disposiciones en toda la Ciudad de México y la firmeza y claridad de la autoridad para hacerlo.

Las diferencias que hay entre ambas legislaciones no las hace opuestas, y al contrario, muestran que la primera es, incluso, más estricta que la segunda respecto del establecimiento de ambientes 100\% libres de humo sin excepción en espacios cerrados, mientras que la segunda reconoce la posibilidad de que se establezcan (bajo estrictas restricciones) zonas exclusivas para fumar (que no es lo mismo que la división tradicional entre espacios compartidos para fumadores y no fumadores) y es mucho más amplia respecto de las regulaciones vinculadas con la importación, etiquetado, empaquetado, publicidad, promoción y venta. Una y otra representan parte del gran esfuerzo sanitario que retoma la protección de bienes globales (como la salud) con medidas locales.

Estos dos instrumentos son, a ciencia cierta, valiosas herramientas para avanzar en la contención de la epidemia de tabaquismo y en el caso de la legislación local, significa un modelo más avanzado y muy ilustrativo de las ventajas que tiene instaurar -sin excepción- am- bientes 100\% libres de humo, como medida universal en todos los espacios públicos cerrados, de ahí que deba considerarse como referencia obligatoria a la que se debe aspirar técnicamente.

Por su parte, lo que resta a la LGCT una vez que sea publicada y que 90 días después entre en vigor en todo el territorio nacional, es avanzar en todos los aspectos regulatorios a reglamentar en materias como importación, empaquetado, etiquetado, promociones, publicidad, regulación de la venta, aplicación de sanciones y multas (de ahí la importancia del reglamento respectivo) e implantar y supervisar lo relativo a los ambientes $100 \%$ libres de humo de tabaco en todos lo espacios públicos cerrados.

La instrumentación de la LGCT, sin embargo, requerirá de una estructura administrativa y jurídica de la que carece el país. Por ello, la Secretaría de Salud -con la colaboración de la Iniciativa Global Bloomberg- ha iniciado el diseño para la construcción de una oficina específica para trabajar en la materia, misma que dependería de la autoridad federal.

Esta instancia se encargará de fortalecer las acciones del Gobierno Federal encaminadas a la aplicación de la Ley y de políticas públicas de acuerdo con los lineamientos del CMCT de la OMS de manera sustentable y permanente. Algunos de sus objetivos son:

- Implementar el CMCT de la OMS y dar cumplimiento a las directrices acordadas por la Conferencia de las partes en tiempo y forma.

- Desarrollar la Estrategia y el Plan de Acción para coadyuvar en el establecimiento de la LGCT y otras disposiciones relativas.

- Definir líneas de acción en la materia para ser implementadas en los ámbitos del Gobierno Federal, estatal y municipal.

- Implantar y dar vigencia a la LGCT en todo el país.

- Reconocimiento de la capacidad instalada de la estructura institucional, recursos disponibles y ámbitos preferenciales de acción.

- Reglamentación de los productos de tabaco: empaquetado y etiquetado; venta; publicidad, promoción y patrocinio de productos del tabaco.

- Seguimiento de las acciones implementadas en relación con el etiquetado, promoción, venta, publicidad, impuestos y espacios 100\% libres de humo.

- Fortalecimiento de la red de servicios de cesación.

- Apoyo a la investigación en materia de comportamiento de la epidemia del tabaquismo en México, prevalencia de adicción a la nicotina, mejores prác- 
ticas para el tratamiento de la adicción a la nicotina, entre otras.

- Vigilancia del comportamiento de la industria.

Se trata, en síntesis, de impulsar una política de salud que fortalezca las acciones para el control del tabaco en México. Luego de muchos años de documentar el daño que a la salud de millones de personas produce el tabaco, el acuerdo mundial de proteger bienes globales como la salud, alienta y hace concebir fundadas esperanzas en las posibilidades que ello tiene al hacer vinculatorios compromisos que buscan proteger el bien más preciados de los pueblos: su salud. En el caso del tabaco en México, estamos en la ruta para lograrlo. Las generaciones actuales y las futuras vivirán mejor y ésta será la mayor contribución que nuestra generación haga para la construcción de un México libre de adicciones y por ello, más sano. 